\title{
AUTONOMOUS SYSTEMS FOR OPERATIONS IN CRITICAL ENVIRONMENTS
}

\author{
Agostino G.Bruzzone ${ }^{1}$, Francesco Longo ${ }^{2}$, \\ Matteo Agresta $^{3}$, Riccardo Di Matteo ${ }^{3}$, Giovanni Luca Maglione ${ }^{3}$ \\ 1 DIME University of Genoa \\ URLwww.itim.unige.it - Email agostino@itim.unige.it \\ 2 MSC-LES UNICAL \\ URLwww.msc-les.org - Email f.longo@unical.it \\ 3 Simulation Team \\ URLwww.simulationteam - Email \{agresta, riccardo.dimatteo, maglione\}@simulationteam.com
}

\begin{abstract}
This paper proposes an environment devoted to simulate the use of autonomous systems in the context of space exploratory missions and operations; this research focuses on supporting engineering of autonomous systems and of their innovative artificial intelligences through interoperable simulation. The proposed approach enables also development of training and educational solutions for use of robots and autonomous systems in space critical environments. The paper addresses different application areas including robotic inventory and warehouse solutions, intelligent space guard systems, drones for supporting extravehicular activities and for managing accidents and health emergencies. The paper investigates the potential of autonomous systems as well as their capability to interoperate with other systems and with humans, especially in critical environments. Finally, the paper presents the existing researches for interoperable simulators devoted to address these challenging topics within Simulation Exploratory Experience initiative.
\end{abstract}

Keywords: Interoperable Simulation, Autonomous Systems, Planet Exploration, Cooperative Missions

\section{INTRODUCTION}

The use of autonomous systems is currently popular in many areas and especially in conditions where safety is critical for humans; indeed, there is a need to define and test operational requirements and technological solution for these systems in different context such as accidents, critical environments and emergency management (Longo 2010; Kawatsuma et al.2012). From this point of view the use of Nano and Micro UAV (Unmanned Aerial Vehicle) and UGV (Unmanned Ground Vehicle) in urban environment is already pretty popular and several researches are on-going on interoperating different systems in order to cover these context over different domains (Mazal et al. 2011;Kuntze et al.2012). These systems are indeed well suited to operate IVA (Intra Vehicular Activity) and EVA (Extra Vehicular Activity) in case of accidents and/or critical conditions. Indeed IVA could be assimilated to indoor operations carried out by different AxS (de Haag et al. 2007; Ulam et al.2010; Chowdhary et al.2012; Rodriguez-Araujo et al. 2014; Tang et al. 2014). The authors have been involved in conducting researches respect these applications for cases of fires or contaminations where to access parts of plants or facilities was a necessity as well as to collect samples or to conduct triage for supporting health assessment and applying elementary medical treatments (Bruzzone et al.2014a; Bruzzone et al. 2015a, 2015b).

In many cases, it becomes critical to substitute remotely controlled vehicles with autonomous systems in order to guarantee success, especially due to lacks in communications related to building infrastructures, electromagnetic interferences and long distance network reliability.

It is evident that these conditions are even further stressed within space operations and related critical situations; in particular the communication latency represents a crucial issue while the hostile environment provides great opportunities for autonomous system application.

This paper proposes a flexible architecture to support the development of new simulators able to interoperate with other systems and to reproduce these scenarios operations. In this paper, applicative examples are provided in reference to different context investigated by the authors for space missions: robotic inventory and warehouse solutions, intelligent space guard systems, drones for supporting extravehicular activities (EVA) and for managing accidents and health emergencies. The proposed approach allows using interoperable simulation for virtual prototyping of new autonomous systems and for testing them while interoperating with other equipment and mission 
crew. This new simulators enable the creation of training and educational framework to promote the use of these innovative autonomous systems.

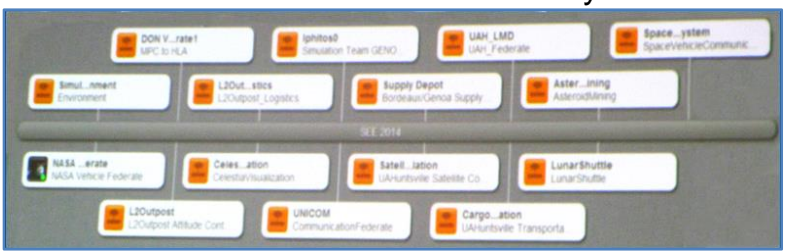

Figure 1 - SEE2014 Federation

The authors plan to integrate these simulators within the initiative Simulation Exploratory Experience (SEE), formerly named Smackdown in reference to mission critical conditions within complex scenarios such as space and planet exploration.

\section{SIMULATION FOR SPACE EXPLORATION}

Considering the flexibility demonstrated by the Intelligent Agents and Virtual Simulators developed within the MS2G (Modeling and interoperable Simulation and Serious Games) paradigm, currently, the authors are working to integrate AxS (Autonomous multidomain System) virtual simulators also in federations applied to Space Exploration (Bruzzone et al.2011; Bruzzone et al.2014b). In particular the authors created in the past different simulators for SEE/Smackdown initiatives including SPIRALS (Space Interoperable Refilling and Advanced Logistics Simulator), IPHITOS (Interoperable simulation of a Protection solution based on light Interceptor Tackler operating in Outer Space) and SISMA (Medical Simulator of Astronaut including treatments, analysis and sickness models). The simulators developed for these purposes adopted HLA IEEE1516 (High Level Architecture) standards and were tested over different RTI (Run Time Infrastructures) since 2011 in Boston, up to 2015 in Arlington. These three simulators developed by Simulation Team were federated with other simulators composing SEE/SmackDown federation as proposed in figure 1; indeed in SEE2014 event the integration testing on SEE federation was conducted in Tampa under supervision of NASA staff (Elfrey \& Waite 2014).

Integration tests on these simulators, within SEE Federation, were conducted both locally and remotely over WAN (Wide Area Network) and presented in the following contexts:

$\begin{array}{cll}\text { Smackdown } & \\ \text { • } 2011 \text { SpringSim / MIT } & \text { Boston } \\ \text { - } 2011 \text { I3M / CAX Forum } & \text { Rome } \\ \text { - } 2012 \text { Springsim } & \text { Orlando } \\ \text { - } 2012 \text { I3M } 2012 \text { CAX Forum } & \text { Wien } \\ \text { - } 2013 \text { Springsim } & \text { Rome } \\ \end{array}$

$\begin{array}{cl}\text { - } 2013 \text { ITEC } & \text { Rome } \\ \text { - } 2013 \text { Summersim } & \text { Toronto } \\ \text { - } 2013 \text { I3M } & \text { Athens } \\ \text { Simulation Exploratory } & \text { Experience } \\ \text { - } 2014 \text { Springsim } & \text { Tampa } \\ \text { - } 2014 \text { I3M } & \text { Bordeaux } \\ \text { - } 2014 \text { CAX Forum } & \text { Istanbul } \\ \text { - } 2015 \text { Springsim } & \text { Arlington } \\ \text { - } 2015 \text { I3M } & \text { Bergeggi }\end{array}$

SEE/Smackdown initiative is led by NASA and sponsored by several companies with the main goal to advance HLA culture by creating a distributed HLA Federation of a Moon Base (Elfrey et al.2011). The initiative is under patronage of Simulation Team and Liophant Simulation. Indeed SEE/Smackdown was originally developed by Priscilla Elfrey and Bill Waite as a competitive environment for students to exploit HLA potential and to diffuse knowledge about interoperable simulation.

The Smackdown/SEE federation is a distributed interoperable simulation that could operates over a WAN through a VPN (Virtual Private Network) interconnecting different locations from NASA Centers, to University Labs all over the world (Elfrey 2013).

\section{STUDENT TEAMS IN SEE/SMACKDOWN}

The Genoa University students, along theses years, developed the Simulation Team Federates proposed in this paper; indeed they operated under the guidance of Prof.Agostino G. Bruzzone (Team Faculty Advisor) and were coordinated by Luciano Dato (Team Leader).

The students applied heavily all their technical and team working skills in order to achieve the final success. In facts, the excellent functionalities of the Tech Forum provided by NASA Technical Staff, as well as Management facilities have drastically supported the collaboration among different Universities and the integration of their federates. In order to collect information to improve the physics of the objects of the simulators the NASA Technical Reports Server (NTRS) database resulted very useful. Starting from this gathered information, realistic conceptual models have been developed, carefully analyzed, implemented, tested and integrated.

Testing HLA in such an interesting and complex initiative is very challenging and NASA technical coordination with support of University Experts is very effective in training the participating teams. In particular it is very interesting for students to face challenges and technical issues related to different problems as well as to the interoperation among the federates. 
Also the contributions by industries result crucial for the success of the initiative; in addition to provide the RTI to the partners several technical contributions are very valuable for the students, including especially the lectures offered by Bjorn Möller on the HLA 1516 standards. By this approach the students acquire know how on the most innovative functionalities offered by HLA and opportunities to test them in challenging contexts. Moreover, working together into a distributed international framework, the teams could improve their capabilities in project management and team working. The Team Leaders reinforce this opportunity through their coordinating and communicating activities during the continuous virtual meeting and distributing testing session along the year. For instance, during the weekly Tag-Up Meetings organized with the purpose to support teams activities each participating units share problems and proposals. The students involved in software development could enhanced their expertise in using JAVA and C\# for HLA simulator by working through object-oriented paradigm in space exploration context. Engineering students devoted to focus on conceptual modelling and problem analysis are learning how to coordinate their work with computer scientists to enhance the capability to Verify, Validate the simulators, to succeed in integration tests, simulation experimentation and data analysis. The development of Simulation Team Federates included strong and extended cooperations; for instance, the Genoa Team has strongly interacted with the Calabria Team in IPHITOS especially with reference to "Asteroid Alert" messages, virtual reality framework and communication. Furthermore, SPIRAL simulator, initially developed by Genoa University, was further implemented in cooperation among Genoa and Bordeaux Universities; during some years the Supply Depot federate was mainly controlled by Genoa students while in other ones was led by Bordeaux Students.

\section{SPACE AUTONOMOUS SYSTEMS SIMULATION}

Space exploration is the ongoing discovery and exploration of celestial structures by the continuous improving of space technologies; so it is evident that in future the exploration missions are expected to establish a solid partnership between human crew and robots (Ambrose et al. 2010). Indeed NASA is already employing robot, telerobots (remotely operated) and Autonomous Systems and draw conclusions that identify critical elements in:

- Technologies enabling Autonomy Capability

- Technologies able to exceed human performance (e.g. sensing, piloting, driving, manipulating, rendezvous and docking).
- Technologies supporting cooperative behaviors among the autonomous systems and humans

- Autonomous Systems increasing human crews independency from Earth Headquarters

Indeed to investigate and develop new solutions able to address decision autonomy and collaborative behavior it is necessary to develop simulators. In facts, there are researches on using simulation for developing, testing and validating technological solutions, algorithms and/or methods for space missions; for instance it was studied the comparison among challenges of space navigation and integration of payload elements in an Unmanned Aerial Vehicle (UAV) for in-flight testing of systems and algorithms (Molina et. al 2014). In this research, UAVs are the key enablers of scientific capabilities to fill up the gap between laboratory simulation and expensive, real space missions.

In other cases, it was underlined how distances affect communications lags present with Earthbased control centers will force to further extend the autonomy capabilities of future human spaceflight (Olofinboba et al. 2005). From this point of view, an intelligent astronaut associate (IAA) has the potential to enhance the ability of astronaut crews to work more efficiently, to cope with uncertainty and to address the unique challenges outlined above. The research describes a preliminary IAA which would provide an intelligent automation interface to the astronaut crew, providing dynamically relevant real-time information, while monitoring cognitive state and workload to best present pertinent information to the astronauts.

Indeed, it could be necessary to develop innovative collaborative performance models able to capture critical performance attributes for supporting human-robotic interface over decisions. The literature as well as the experimentation outlines the importance of trustiness in human-robot relationship; for instance some researches confirm these aspects through a decision-analytical based measure of trust tested over experiments related to human-robot collaborative task in MITPAS (mixed initiative team performance assessment system) simulation environment (Freddy et al. 2007).

Space robotics is also considered one of the most promising approaches for on-orbit servicing (OOS) missions such as docking, berthing, refueling, repairing, upgrading, transporting, rescuing, and orbital debris removal.

In this area, there are interesting extensive surveys on use of space telerobots and related concepts of operations for future space exploration missions (Fong et al. 2013).

Obviously the success of these researches and their applicative potential rely on enabling techniques; from this point of view even if several manned on-orbit servicing missions were successfully accomplished, no unmanned, fully autonomous missions have not been done yet. 
Furthermore, all previous unmanned technology demonstrators were designed to address docking and rendez-vous only with cooperative targets; vice versa non-cooperative satellites still represent an open issue.
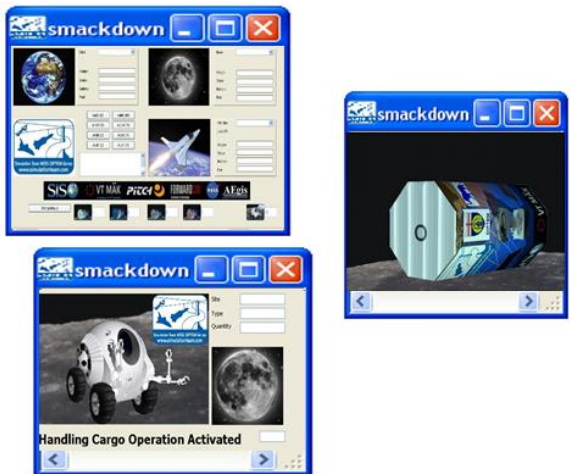

Figure 2 - SPIRALS GUI

Therefore, the use of autonomous systems could support the improvement of safety and reliability during docking space targets (e.g. spacecraft or satellites); this aspect becomes even more important if the target has an unknown motion and kinematics/dynamics properties. Indeed, there are interesting state of arts also on the enabling technologies devoted to support these aspects including kinematics, dynamics, control and verification of space robotic systems in manned and unmanned on-orbit servicing missions (Flores et al. 2015).

The deployment of teleoperated space robots for servicing and maintenance operations is a valuable addition to existing autonomous systems, because it will provide flexibility and robustness in mission operations even if its capabilities could be limited in case of deep space exploration due to communication constraints. Researches on robotic swarm and experiments for these purposes has been conducted in reference to operation controlled by astronauts aboard the International Space Station (ISS). The Synchronized Position Hold Engage Reorient Experimental Satellites (SPHERES) have been studied in reference to space operations (Stoll et al 2015).

Autonomous systems could adopt autonomous landing navigation and guidance capabilities for future missions on asteroid devoted to collect samples (Li et al 2015).

Indeed, there are researches devoted to develop autonomous servicing spacecraft simulator to be used as test-bed for validation of autonomous control algorithms (Spencer 2001).

\section{SIMULATION TEAM HLA SPACE SIMULATORS}

Therefore, in this paper the innovative idea is to create an AXS HLA simulator able to be federated with other simulators and real systems. This approach allows to simulate use of AxS in space operations within an exploratory mission for managing critical conditions (e.g. space protection from debris and small asteroids, accidents, base atmosphere contaminations, astronaut sickness) as well as regular activities (e.g. warehouse loading/unloading, supporting EVA operations).

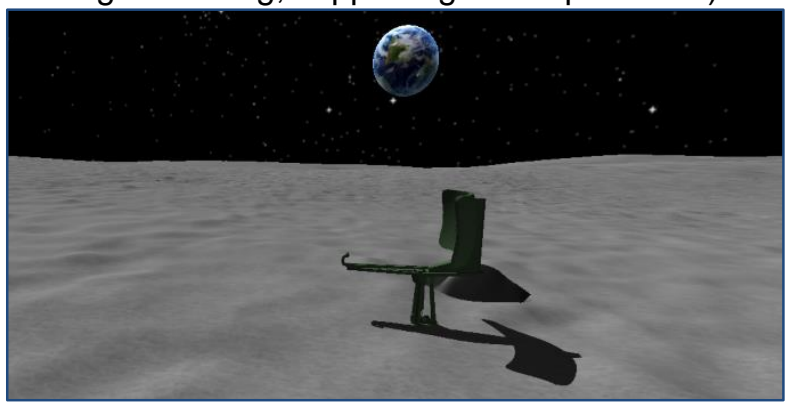

Figure 3 - IPHITOS Spaceguard Controller

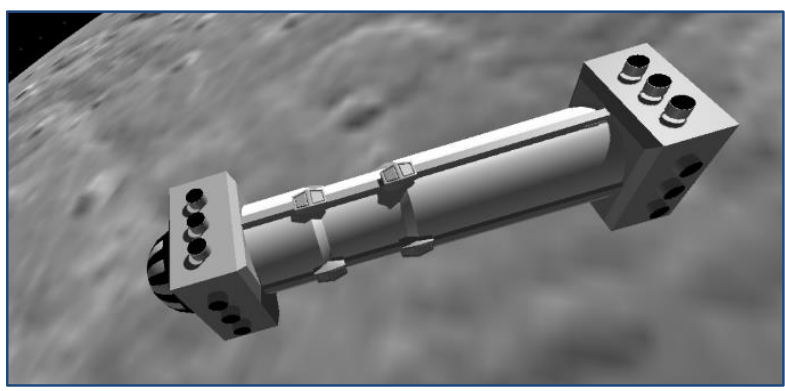

Figure 4 - IPHITOS Interceptor missile over the Moon after exhausting main propulsion phase

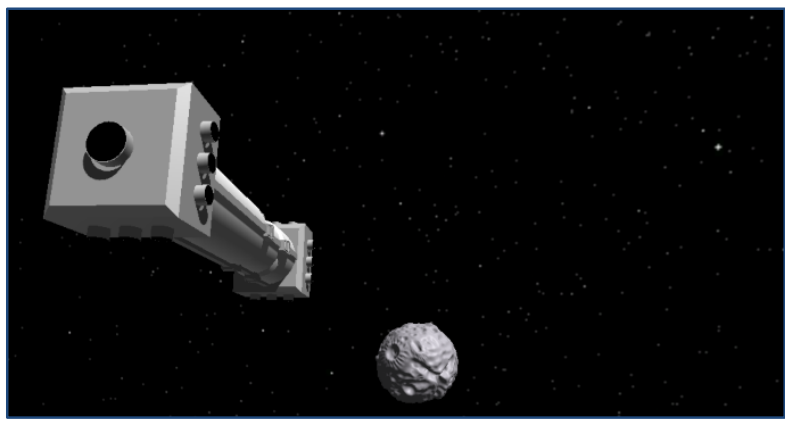

Figure 5-IPHITOS Interceptor heading to the asteroid for deviating it

The proposed simulators have been integrated and tested within SEE/Smackdown. In these cases the autonomous systems should be able to assess situation awareness by sensor input and through further investigations; the AxS will be able to move, navigate or send other entities as well as to interact with other systems. AxS through interactions with other equipment, systems and even with mission crew will conduct tasks, apply treatments and complete actions devoted to guarantee mission success (Dautenhahn 2007). Indeed the AxS should have the flexibility to move, interact and inspect different areas of the base/spacecraft, to collect informations, samples and measures about the situation as well as to help personnel confined in these areas. These simulators could be used to conduct operations inside the base as well as EVA for inspections and checks outside the 
base/spacecraft; obviously, the autonomous systems devoted to the different tasks could be different and specific, even if flexibility and modularity could be precious characteristics in long terms space explorations.

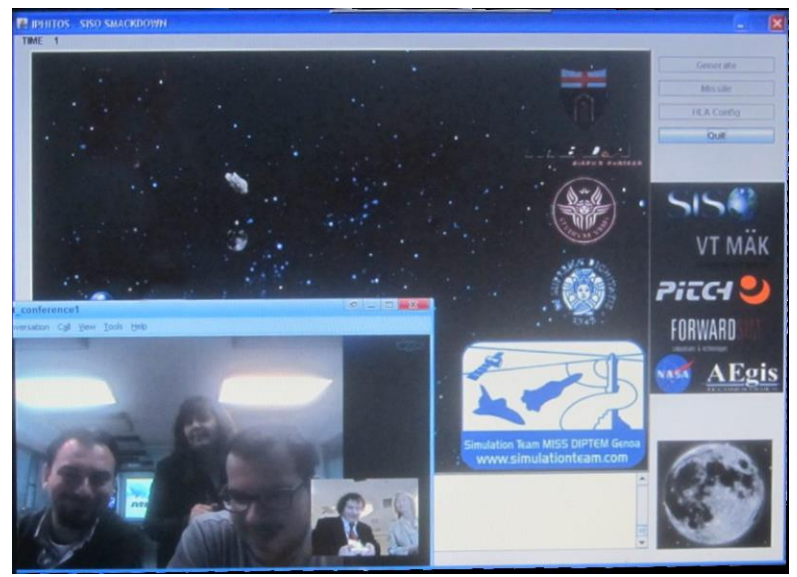

Figure 6 - IPHITOS Running over the Web from Italy to Florida federated in Smackdown 2013

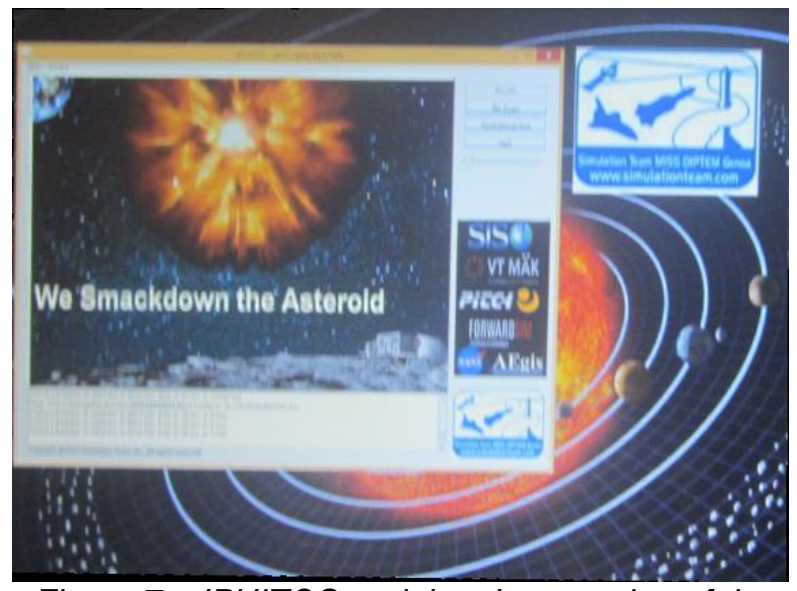

Figure 7-IPHITOS real time Interception of the Asteroid in San Diego during SEE2014

This approach is useful to reproduce both regular activities and mission critical scenarios.

\section{SPIRALS}

SPIRALS Simulator was developed by students of Genoa University and extended to Bordeaux University students; the team was including people in Internship in MAST with patronage of Liophant Simulation (Dato \& Dallorto 2013). SPIRALS federate was in charge of the inventory management, operations \& logistics for the Moon Base. SPIRALS was including since the beginning Agents devoted to Cargo Handling Operation supposed to be able to load/unload the shuttle as well as the Moon base Depots. Autonomous Vehicle were used for handling materials in the storage room without too much concerns about their mechanical configuration, therefore in future models these aspects could be considered; similar issues affects goods storage boxes and containers. The model originally included the possibility to associate the depots to any object; each depot includes the inventory management systems, the drones devoted to cargo handling as well as storage systems and reliability models for each equipment and good.

Originally there where three main locations: Earth, Moon base and shuttle; the goods include Oxygen, Water, Food, Batteries, Fuel as well as $\mathrm{He} 3$.

\section{IPHITOS}

IPHITOS is the Moon Base protector against threats such as space debris and small asteroid. The simulator was originally developed in 2013 by Simulation Team in cooperation with students of Genoa University, La Sapienza and in Internship in MBDA and MAST. IPHITOS simulator was renewed in 2014 by Simulation Team with students in internship with MAST. The simulator reproduces both the threat represented by an asteroid as well as the spaceguard system and the interceptors; the system interoperate with satellite network, moon base, shuttle and other objects transmitting updates about the space threat and related countermeasures. Intelligent Agents are dedicated to identify the assets and the best approach to deviate them through use of interceptors as well as to provide informations to other federates. IPHITOS sends messages through HLA Interactions and updates on collision estimations as well as dangerous areas on the surface for supporting escape of units performing an EVA.

While the Moon follows its orbit around the Earth, the asteroid could be detected and tracked in terms of position and velocity continuously monitored by the Spaceguard Systems. When the boundary conditions guarantees the possibility to intercept and deviate or destroy the space threat the IPHITOS interceptor is launched towards its target. IPHITOS could run fast time or slow time and considers the influence of different gravitational fields (e.g. Earth, Sun and Moon).

The Intelligent Spaceguard is in charge to analyze the situation and use Satellite Network to communicate with the different assets, while the most appropriate actions should be identified to engage the specific space threat. The simulator is time constrained and time regulating running fast time and real time based on user preferences and federation settings. Original version was including Matlab models for the interceptor and $\mathrm{C}++$ code, while current release is fully implemented in Java.

\section{SISMA}

SISMA is a simulator taking care of astronaut health status and possible analysis and elementary medical treatment. The astronaut could be associated to any location and/or object such as the Moon Base or the shuttle; the association introduces physiological issues (e.g. acceleration, gravity, stress). SISMA includes constant monitoring biometrics as well as possible medical analysis to be carried out on demand. In the 
simulator the data are collected by wearable sensors and could be integrated with additional actions performed by autonomous vehicles.

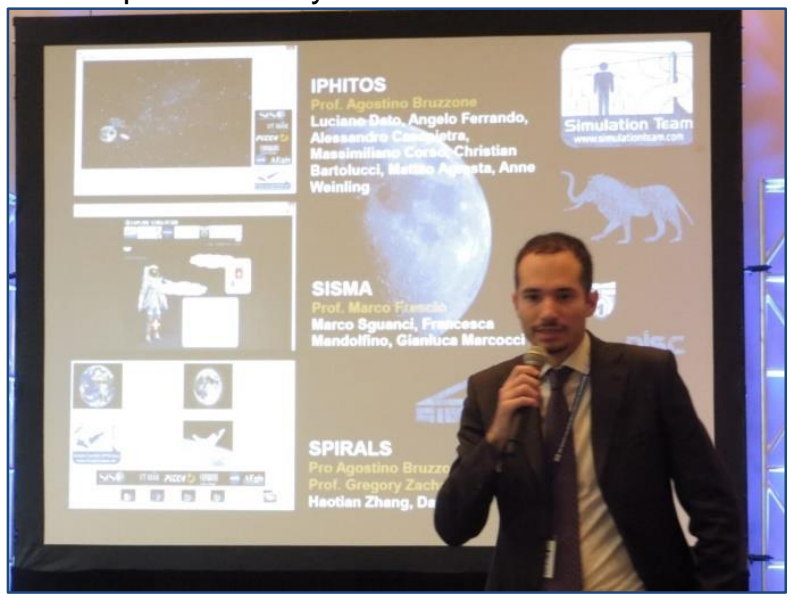

Figure 8-Dato Presenting SISMA and its HLA

Gateway for interacting with the web application

The drones could conduct direct analysis or additional sampling and test for this purpose and for triage in case of accidents. If sickness and/or symptoms emerge and affect the Astronaut's capabilities; it is possible to suggest analysis and treatment that could be eventually managed by the autonomous systems. The simulation considers critical conditions represented by threshold levels of Astronaut's health parameters. This simulator was mixing engineering, computer science and medical profiles (Prof. Bruzzone \& Frascio supervisors, Dato \& Sguanci as Team Leader, Ferrando, Mandolfino, Marcocci as students).

Technical and teamworking skills have been the key for each Team member to develop an effective conceptual model. After having studied several scientific works about simulation in the medical field and characteristic problems related to life in space, the Team has chosen some internist pathologies (not surgical ones) to be modelled: Asthma, Renal Calculosis and Space Adaptation Syndrome (SAS). The simulator reproduces the data collection systems devoted to support the patient analysis, diagnosis and treatments of each case. More in details, once symptoms have been analyzed, doctors on Earth finalize the diagnosis and suggest a treatment. In case the astronaut is in critical conditions, the autonomous system could approach him and perform direct measures, assess the situation and apply treatment. First basic action of the drone is to conduct a triage and to identify the situation of the critical space patient. Currently the authors are considering to add pathologies related to accidents and critical conditions on the base/spacecraft (e.g. pressure loss, atmosphere contamination, traumatic events). Blood and other laboratory tests could be carried on samples in order to refine the initial diagnosis and focus the related clinical treatment to be taken. The simulator is expected to monitor the situation, considering also the possibility for the pathology to evolve in different directions, till the moment of the complete healing of the Astronaut or the impossibility to carry out more specific operations within the spacecraft/base.

SISMA conceptual model and implementation have been developed jointly by the mix team including computer scientists, engineers and medical doctors. The SISMA medical assistance simulator has been created by using the MVC4 (Model View Controller) Architecture, a technology used to develop web applications. The related web page has been defined by HTML5 Markup language and it is designed to be activated by Google Chrome browser. The adoption of the C\# language (Object Oriented) to implement the logic of the simulator (server side) has involved some issues related to the fact that this language does not support directly High Level Architecture. The team proposed a solution based on the creation of a Java program able to manage (thanks to socket TCP) all the communications between HLA and C\# coded program. However, another issue related to the different encoding for the socket communication used by Java and C\# had to be faced and it was successfully solved by the team. The animations were implemented through a JQuery library since, using Javascript language, it is possible to save time and simplify the developments. The first release of SISMA was a challenge considering the trans-disciplinary nature of the team; therefore they were very motivated and resulted able to finalize the development of this medical field simulator for a Space Federation. Moreover the team capabilities benefit of the experience in applying innovative M\&S skills over distributed interoperable simulation frameworks as well as the interactions with other international teams and supervisors.

\section{EVAD}

EVAD (Extra Vehicular Activities And Drones) A new simulator is under development to reproduce EVA activities and the support provided by autonomous systems; the idea is inspired by the activities carried out underwater by using AUV (Autonomous Underwater Vehicles) and ROV (Remoted Operated Vehicles) for supporting scuba divers in Oil and Gas platforms as well as in Mine Countermeasures (Bruzzone et al 2015d).

The drones in this context could be devoted to multiple tasks such as monitor the astronaut, to support its activities (e.g. bringing tools) and to act as first responder in case of accidents or emergencies. In all these tasks, the autonomous systems should interact with other systems and equipment, putting into evidence the importance to create interoperable simulation to properly design the new systems and define their procedures.

Indeed the authors developed an interactive interoperable virtual CAVE (Cave Automatic Virtual Environment) named SPIDER (Simulation Practical Immersive Dynamic Environment for 
Reengineering) to understand the complex context where multiple AxS interoperates and to investigate new solutions to assign orders and joint collaborative tasks. Indeed, it is possible to control AxS also in space respect its interaction with other assets as well as astronauts in critical missions or during emergencies.

\section{CONCLUSIONS}

This paper proposes an integrated approach based on HLA architecture that allows to virtual prototype innovative autonomous systems supporting space and exploratory missions.

This approach enables also the integration of real equipment as well as different virtual simulators to conduct tests and experimentations.

The proposed research was integrated within Smackdown/SEE initiative and demonstrated over multiple sites and along last years. Currently the authors are developing simulators addressing specific applications to be federated within this context. This approach was applied effectively to several cases in other frameworks such as industrial and defense applications related to emergencies and disasters with special attention to complex environments and operations in external and internal activities within bases/spacecrafts.

Currently the authors are developing new extensions for these simulators in order to address Space Explorations and additional challenges such as decision autonomy and communication latencies. This approach based on HLA standards and consolidated architectures guarantees reusability with low efforts; indeed the authors will conduct test on new evolutions of the simulators proposed in this paper during new Simulation Exploratory Experiences.

\section{REFERENCES}

- Ambrose R., Brian Wilcox, Ben Reed, Larry Matthies, Dave Lavery, Dave Korsmeyer (2010) "Robotics, Tele-Robotics and Autonomous Systems Roadmap", NASA Roadmap Technology Area 04, Washington DC, November

- Bruzzone A.G., Marina Massei, Matteo Agresta, Alberto Tremori, Francesco Longo, Giuseppina Murino, Fabio De Felice, Antonella Petrillo (2015a) "Human behavior simulation for smart decision making in emergency prevention and mitigation within urban \& industrial environments", Proc. of I3M, Bergeggi, September

- Bruzzone A.G., Marina Massei, Alberto Tremori, Diego Crespo Pereira, Giulio Franzinetti, Manlio Oddone, Arnau Carrera, Fabio Camponeschi, Luciano Dato (2015b) "Autonomous system simulation to improve scenario awareness and capabilities to protect marine, off-shore and coastal critical infrastructure", Proceedings of I3M, Bergeggi, Italy, September

- Bruzzone A.G, Longo F., Nicoletti L.C. (2015c) "Multidisciplinary Approach to Disaster Management", Proceedings of Summer Computer Simulation Conference, SCS, Chicago, IL, July

- Bruzzone A.G., Marina Massei, Alberto Tremori, Diego Crespo Pereira, Giulio Franzinetti, Manlio Oddone, Arnau Carrera, Fabio Camponeschi, Luciano Dato (2015d) "Autonomous System Simulation to improve Scenario Awareness and Capabilities to Protect Marine, Off-shore and Coastal Critical Infrastructure", Proceedings of WAMS2015, Bergeggi, Italy, September

- Bruzzone A.G., Marco Frascio, Francesco Longo, Alessandro Chiurco, Simone Zanoni, Lucio Zavanella, Paolo Fadda, Gianfranco Fancello, Domenico Falcone, Fabio De Felice, Antonella Petrillo, Pasquale Carotenuto (2014a) "Disaster and emergency management simulation in industrial plants", Proceedings of European M\&S Symposium, Bordeaux, France, September

- Bruzzone A.G., Marina Massei, Alberto Tremori, Francesco Longo, Letizia Nicoletti, Simonluca Poggi, Christian Bartolucci, Edoardo Picco, Guglielmo Poggio (2014b) "MS2G: simulation as a service for data mining and crowd sourcing in vulnerability Reduction" Proceedings of WAMS2014, Istanbul, Turkey, September 16-19

- Bruzzone, A.G., Tremori, A., Massei, M., (2011) "Adding Smart to the Mix," Modeling, Simulation \& Training: the International Defence Training Journal, 3, 25-27.

- Chowdhary, G., Sobers, D. M., Pravitra, C., Christmann, C., Wu, A., Hashimoto, H., ... \& Johnson, E. N. (2012). Self-contained autonomous indoor flight with ranging sensor navigation. Journal of Guidance, Control, and Dynamics, 35(6), 1843-1854.

- Dato L., Dallorto M. (2014) "Smackdown”, Invited Speech at ITEC2013 Demonstration within MIMOS Booth, Rome, Italy, May

- Dautenhahn, K. (2007). Methodology and themes of human-robot interaction: a growing research field. International Journal of Advanced Robotic Systems.

- de Haag, M. U., Venable, D., \& Smearcheck, M. (2007, April). Use of 3D laser radar for navigation of unmanned aerial and ground vehicles in urban and indoor environments. In Defense and Security Symposium (pp. 65500C-65500C). International Society for Optics and Photonics.

- Elfrey P. and Waite B. (2014) "Simulation Exploratory Experience", Keynote Speech at SEE2014 Closing Ceremony, Tampa, FL, April

- Elfrey P. (2013) "SimSmackdown", Key Note Presentation at Springsim 2013, San Diego, CA

- Elfrey, P. R., Zacharewicz, G., \& Ni, M. (2011). SMACKDOWN: adventures in simulation standards and interoperability. In Proceedings of 
the Winter Simulation Conference, December, pp. 3963-3967

- Flores-Abad, A., Ma, O., Pham, K., \& Ulrich, S. (2014). A review of space robotics technologies for on-orbit servicing. Progress in Aerospace Sciences, 68, 1-26.

- Fong, T., Zumbado, J. R., Currie, N., Mishkin, A., \& Akin, D. L. (2013). Space Telerobotics Unique Challenges to Human-Robot Collaboration in Space. Reviews of Human Factors and Ergonomics, 9(1), 6-56.

- Freedy, A., DeVisser, E., Weltman, G., \& Coeyman, N. (2007, May). Measurement of trust in human-robot collaboration. In Collaborative Technologies and Systems, 2007. CTS 2007. International Symposium on (pp. 106-114). IEEE.

- Kawatsuma, S., Fukushima, M., \& Okada, T. (2012). Emergency response by robots to Fukushima-Daiichi accident: summary and lessons learned. Industrial Robot: An International Journal, 39(5), 428-435

- Kuntze, H., Frey, C. W., Tchouchenkov, I., Staehle, B., Rome, E., Pfeiffer, K., \& Wollenstein, J. (2012) "SENEKA-sensor network with mobile robots for disaster management", Proceedings of IEEE Conference on Homeland Security Technologies (HST), Waltham, MA, November, pp. 406-410

- Li, S., Cui, P., \& Cui, H. (2006). Autonomous navigation and guidance for landing on asteroids. Aerospace science and technology, 10(3), 239247.

- Longo, F. (2010). Emergency simulation: state of the art and future research guidelines. SCS M\&S Magazine, 1(4), 2010-04.

- Macauley, M. K. (2005). Flying in the face of uncertainty: Human risk in space activities. Chi. J. Int'I L., 6, 131.

- Mazal, J., Stodola, P., \& Mokrá, I. (2011) “Tactical and Autonomous UGV Navigation" In Mechatronics Springer Berlin Heidelberg, pp. 439-443

- Molina, P., Angelats, E., Colomina, I., Latorre, A., Montaño, J., \& Wis, M. (2014). The Perigeo Project: Inertial and Imaging Sensors Processing, Integration and Validation on Uav Platforms for Space Navigation. The International Archives of Photogrammetry, Remote Sensing and Spatial Information Sciences, 40(3), 79.

- Olofinboba, O., \& Dorneich, M. (2005). Intelligent Astronaut Associate for Next Generation Space Systems. In Aerospace Conference, 2005 IEEE, pp. 1-7, March

- Rodriguez-Araujo, J., Rodriguez-Andina, J. J., Farina, J., \& Chow, M. Y. (2014). FieldProgrammable System-on-Chip for Localization of UGVs in an Indoor iSpace. Industrial Informatics, IEEE Transactions on, 10(2), pp. 1033-1043.

- Spencer, M. G. (2001). Development of a servicing satellite simulator. In Proceedings of the
AIAA space conference and exposition, No. 2001-4529, pp. 28-30, August

- Stoll, E., Jaekel, S., Katz, J., Saenz-Otero, A., \& Varatharajoo, R. (2012). SPHERES interactHuman-machine interaction aboard the International Space Station. Journal of Field Robotics, 29(4), 554-575.

- Tang, J., Chen, Y., Jaakkola, A., Liu, J., Hyyppä, J., \& Hyyppä, H. (2014). NAVIS-An UGV Indoor Positioning System Using Laser Scan Matching for Large-Area Real-Time Applications. Sensors, 14(7), 11805-11824.

- Ulam, P. D., Kira, Z., Arkin, R. C., \& Collins, T. R. (2010, April). Mission specification and control for unmanned aerial and ground vehicles for indoor target discovery and tracking. In SPIE Defense, Security, and Sensing, International Society for Optics and Photonics 